by ELISA. Inhibitors of RANKL were then tested in vitro. We selected several RANKL inhibitors: anti-RANKL antibody, RANK-Fc, Isoliquiritigenin and Gallocatechin gallate. The efficacy of these inhibitors was indirectly evaluated with the murine macrophage RAW264.7 cell line which undergoes, in the presence of RANKL, an osteoclast differentiation (TRAP and Cathepsin $\mathrm{K}$ expression). The efficacy of RANKL inhibitors was then evaluated, in this model, by RT-qPCR. Apoptosis and proliferation of the cancer cell lines in the presence of the inhibitors were also analyzed.

Results RANKL/PD-L1 expression profile on specimens from each breast cancer subtypes showed that both immunosuppressive molecules are expressed by all breast cancers with a significantly more intense immunoreactivity for triple negative breast cancers. Most of the cell lines expressed both proteins. We found that RANKL is secreted in their extracellular environment. RANKL inhibitors are efficient and will be tested in vivo.

Conclusions Several murine triple negative breast cancer cell lines will be sub-cutaneously injected in mice and the efficacy of both RANKL and PD-L1 inhibitors will be evaluated (separately or in combination). The infiltration of tumor microenvironment by different immune cell populations, the presence of metastasis and the tumor growth will be analyzed.

Disclosure Information C. Pilard: None. P. Roncarati: None. E. Hendrick: None. A. Lebeau: None. D. Bruyère: None. T. Lerho: None. M. Ancion: None. C. Reynders: None. P. Delvenne: None. M. Herfs: None. P. Hubert: None.

\section{P08.04 NEOADJUVANT CHEMORADIOTHERAPY WITH SEQUENTIAL IPILIMUMAB AND NIVOLUMAB IN RECTAL CANCER (CHINOREC): A PROSPECTIVE RANDOMIZED, OPEN-LABEL, MULTICENTER, PHASE II CLINICAL TRIAL}

\begin{abstract}
1,2J Laengle*, 'I Kuehrer, 'D Pils, '1 Kabiljo, ${ }^{3} \mathrm{~K}$ Wöran, ${ }^{3} \mathrm{~J}$ Stift, ${ }^{4} \mathrm{~F}$ Herbst, ${ }^{4} \mathrm{~B}$ Dauser, ${ }^{5} \mathrm{M}$ Monschein, ${ }^{5} \mathrm{P}$ Razek, ${ }^{6} \mathrm{~S}$ Haegele, ${ }^{7} \mathrm{M}$ Herac, ${ }^{7} \mathrm{~W}$ Hulla, ${ }^{8} \mathrm{C}$ Bitterman, ${ }^{8} \mathrm{~F}$ Laengle, ${ }^{9} \mathrm{D}$ Tamandl, ${ }^{10} \mathrm{~J}$ Widder, ${ }^{10} \mathrm{R}$ Schmid, ${ }^{1,2} \mathrm{M}$ Bergmann. ${ }^{1}$ Division of General Surgery, Department of Surgery, Comprehensive Cancer Center Vienna, Medical University of Vienna, Vienna, Austria; ${ }^{2}$ Ludwig Boltzmann Institute Applied Diagnostics, Medical University of Vienna, Vienna, Austria; ${ }^{3}$ Department of Pathology, Comprehensive Cancer Center Vienna, Medical University of Vienna, Vienna, Austria; ${ }^{4}$ Department of Surgery, Hospital of St. John of God, Vienna, Austria; ${ }^{5}$ Department of Surgery, Clinic Floridsdorf, Vienna, Austria; ${ }^{6}$ Department of Pathology, Clinic Floridsdorf, Vienna, Austria; Institute of Pathology, State Hospital Wiener Neustadt, Wiener Neustadt, Austria; ${ }^{8}$ Department of Surgery, State Hospital Wiener Neustadt, Wiener Neustadt, Austria; ${ }^{9}$ Division of General and Pediatric Radiology, Department of Biomedical Imaging and Image-guided Therapy, Comprehensive Cancer Center Vienna, Medical University of Vienna, Vienna, Austria; ${ }^{10}$ Department of Radiotherapy, Comprehensive Cancer Center Vienna, Medical University of Vienna, Vienna, Austria
\end{abstract}

10.1136/jitc-2020-ITOC7.100

Background Immune checkpoint inhibitors (ICI), such as ipilimumab (anti-cytotoxic T-lymphocyte-associated protein 4; CTLA-4) or nivolumab (anti-programmed cell death protein 1 ; PD-1) have been proven to be an effective strategy in solid cancers. Nevertheless, ICI seem not to be effective in microsatellite stable (MSS) tumors, as those potentially lack an immunogenic priming. Radiotherapy is capable to induce an immunogenic cell death (ICD) and subsequently an immunogenic tumor immune microenvironment (TIME). Thus, the pro-inflammatory effect of radiotherapy might restore the susceptibility of MSS tumors to ICI, leading to more pronounced tumor shrinkage, as well as to an effective antitumor immune response.

Material and Methods In total 80 patients with a locally advanced rectal cancer (LARC) will be randomly assigned in a 30:50 ratio, to receive either standard of care (SOC) neoadjuvant chemoradiotherapy alone (CRT; 50 Gy in 2 Gy fractions with capecitabine $1650 \mathrm{mg} / \mathrm{m}^{2} / \mathrm{d}$ over 25 working days) or concomitant with a single dose of ipilimumab 1 $\mathrm{mg} / \mathrm{kg}$ on day 7 following sequentially 3 cycles of nivolu$\mathrm{mab} 3 \mathrm{mg} / \mathrm{kg}$ Q2W starting on day 14. Patients will undergo surgery within 10 to 12 weeks post CRT. The primary endpoint is safety, tolerability and feasibility assessed by the latest Clavien-Dindo classification of surgical complications and the common terminology criteria of adverse events (CTCAE).

Results ClinicalTrials. gov identifier: NCT04124601. Serial liquid (plasma, serum, peripheral blood mononuclear cells) and tissue biopsies will be taken sequentially before, during and after neoadjuvant therapy. Secondary objectives are radiographic (mrTRG) and pathological (TRG) therapy response. Immune cell infiltrate of resected specimen, as well as genomic, transcriptomic, epigenomic and proteomic pattern of sequential liquid and tissue biopsies will be correlated with therapy response and clinical outcome.

Conclusion This is the first in human study, which uses neoadjuvant CRT in LARC patients with concomitant ipilimumab and nivolumab, applied in a sequential approach. A detailed understanding of therapy induced changes during neoadjuvant CRT with concomitant ICI in a human translation setting will allow the application of radiotherapy as a part of novel immunotherapeutic concept. This is an investigator-initiated trial (IIT), which received a research grant and the study medications from Bristol-Myers Squibb (BMS).

Disclosure Information J. Laengle: B. Research Grant (principal investigator, collaborator or consultant and pending grants as well as grants already received); Significant; IIT, Research Grant \& Study Medication from BMS. I. Kuehrer: None. D. Pils: None. J. Kabiljo: None. K. Wöran: None. J. Stift: None. F. Herbst: None. B. Dauser: None. M. Monschein: None. P. Razek: None. S. Haegele: None. M. Herac: None. W. Hulla: None. C. Bitterman: None. F. Laengle: None. D. Tamandl: None. J. Widder: None. R. Schmid: None. M. Bergmann: B. Research Grant (principal investigator, collaborator or consultant and pending grants as well as grants already received); Significant; IIT, Research Grant \& Study Medication from BMS.

\section{P09 Young Researchers Session}

\section{P09.01 ADOPTIVE CELL THERAPY OF HEMATOLOGICAL MALIGNANCIES USING CYTOKINE-INDUCED KILLER CELLS RETARGETED WITH MONOCLONAL ANTIBODIES}

${ }^{1} \mathrm{~A}$ Dalla Pietà*, 'E Cappuzzello, ${ }^{1} \mathrm{P}$ Palmerini, ${ }^{2} \mathrm{R}$ Sommaggio, ${ }^{3} \mathrm{G}$ Astori, ${ }^{3} \mathrm{~K}$ Chieregato, ${ }^{4} \mathrm{O}$ Perbellini, ${ }^{4} \mathrm{M}$ Tisi, ${ }^{5} \mathrm{C}$ Visco, ${ }^{4} \mathrm{M}$ Ruggeri, ${ }^{1,2} \mathrm{~A}$ Rosato. ${ }^{1}$ Department of Surgery, Oncology and Gastroenterology, Immunology and Oncology Section, University of Padua, Padua, Italy; ${ }^{2}$ Veneto Institute of Oncology IOV - IRCCS, Padua, Italy; ${ }^{3}$ Advanced Cellular Therapy Laboratory, Hematology Department, San Bortolo Hospital, Vicenza, Italy; ${ }^{4}$ Hematology Department, San Bortolo Hospital, Vicenza, Italy; ${ }^{5}$ Department of Medicine, Section of Hematology, University of Verona, Verona, Italy

10.1136/jitc-2020-ITOC7.101 